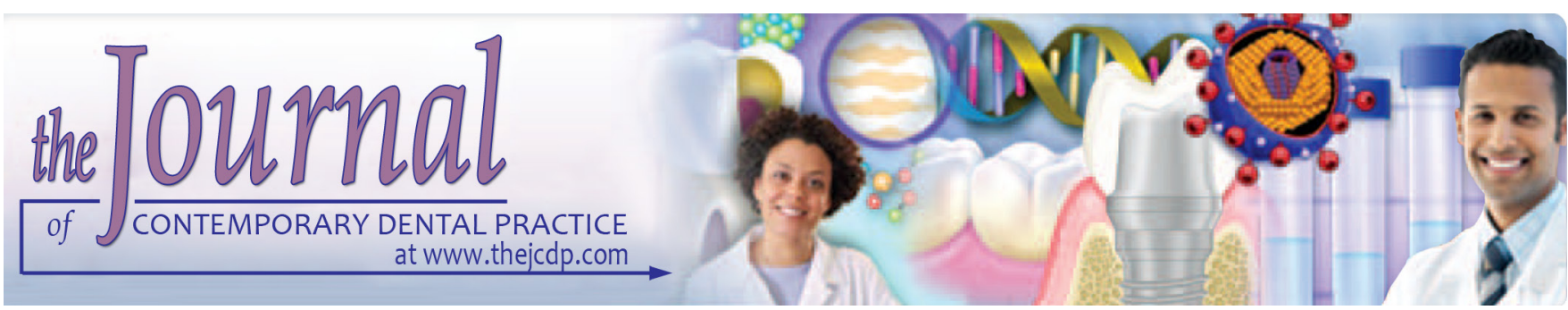

\title{
Comparison of Plaque Removal and Wear between Charcoal Infused Bristle and Nylon Bristle Toothbrushes: A Randomized Clinical Crossover Study
}

\author{
${ }^{1}$ Vineet Kini, ${ }^{2}$ Seema Yadav, ${ }^{3}$ Jaishree A Rijhwani, ${ }^{4}$ Arjumand Farooqui, ${ }^{5}$ Amruta A Joshi, ${ }^{6}$ Sidheshwar G Phad
}

\begin{abstract}
Aim: To compare plaque removal and wear between charcoal infused bristle toothbrushes (T1) and nylon bristle toothbrushes (T2) in a randomized clinical crossover study.

Materials and methods: A cross-over study was conducted in 2 phases of 6 weeks duration each with an intervening 2-week washout. Twenty-five participants meeting inclusion criteria were randomly allocated into groups A (13) and B (12). In phase 1: group $A$ was assigned T1 and group $B$ was assigned T2. Toothbrushing was advised twice daily for 2 minutes by modified bass technique after meals. At baseline, 3 weeks and 6 weeks the wear index $(\mathrm{WI})$, plaque index $(\mathrm{PI})$ and gingival index $(\mathrm{Gl})$ were recorded. Following washout in phase 2 group A was assigned T2 and group B was assigned T1 and the same study protocol was followed.
\end{abstract}

Results: Intra-group comparison between baseline, 3 and 6 weeks by the paired t-test resulted in significant reduction in $\mathrm{PI}, \mathrm{GI}$ and increase in WI $(p<0.05)$ for T1 and T2. Inter-group comparison using the unpaired t-test resulted in WI for T1 being significantly higher $(p<0.05)$ at 3 weeks and lower at 6 weeks $(p<0.05)$ compared to T2. PI for T1 was significantly higher at 3 weeks $(p<0.05)$ and lower at 6 weeks $(p<0.05)$ compared to T2. No significant difference in GI scores between T1 and T2 at 3 and 6 weeks was observed $(p>0.05)$.

Conclusion: Charcoal infused bristles demonstrated less wear and more plaque removal compared to nylon bristles.

Clinical significance: Charcoal infused bristles demonstrate less wear compared to nylon bristles.

1,3-6 Department of Periodontics, MGM Dental College and Hospital, Mumbai, Maharashtra, India

${ }^{2}$ Department of Periodontics, Faculty of Dentistry, SEGi University, Kota Damansara, Malaysia

Corresponding Author: Vineet Kini, Department of Periodontics, MGM Dental College and Hospital, Mumbai, Maharashtra, India, Phone: +919769804390, e-mail: drvinkin@gmail.com
Keywords: Charcoal infused bristle, Cross-over study, Nylon bristle, Plaque removal, Randomized clinical trial, Toothbrush wear.

How to cite this article: Kini V, Yadav S, Rijhwani JA, Farooqui A, Joshi AA, Phad SG. Comparison of Plaque Removal and Wear between Charcoal Infused Bristle and Nylon Bristle Toothbrushes: A Randomized Clinical Crossover Study. J Contemp Dent Pract 2019;20(3):377-384.

Source of support: Nil

Conflict of interest: None

\section{INTRODUCTION}

Plaque control measures are essential to maintain periodontal health by preventing the accumulation of dental plaque. ${ }^{1}$ Routine mechanical plaque control measures comprise use of manual toothbrushes with dentifrices. The efficacy of mechanical plaque control with a manual toothbrush depends on user dexterity, technique, force, duration, and frequency during brushing. ${ }^{2-5}$ Based on individual preference and oral hygiene needs, the toothbrush design is an important aspect to consider. ${ }^{6-8}$ The toothbrush design is dependent upon toothbrush head size, shank angulation, bristle material, and their arrangement. The bristle length and angulation determine the brushing plane of toothbrushes. ${ }^{9-11}$ The nylon bristle toothbrush is commonly used with varying bristle diameter, length, taper, and arrangement within tuft slots in the head of the toothbrush. ${ }^{2,6,8,11}$ The diameter of the bristle filament determines the bristle flexibility and the texture of the toothbrush into hard, medium, soft and ultra-soft. ${ }^{2,9}$ Nylon bristles undergo flaring or splaying leading to toothbrush wear depending on brushing force, frequency, and duration on use..$^{2-5}$ Improper brushing technique and malalignment of teeth also contribute to bristle wear. The bending, splaying and flaring of worn bristles possibly 
affect their plaque removal efficacy requiring replacement of toothbrushes. ${ }^{2,12-14}$ The accessibility of the toothbrush bristles to remove plaque from the tooth surfaces along the gingival margin depend on the bristle diameter and on whether bristle tips are rounded or tapered. ${ }^{15-17}$ Recently charcoal infused nylon bristle toothbrushes have been introduced in addition to the already available nylon bristle toothbrushes. This study was conducted to determine whether if charcoal infused nylon bristles show any difference in bristle wear and plaque removal efficacy compared to regular nylon bristles of the same dimension following use over a period of time keeping other parameters that influence oral hygiene similar.

\section{MATERIALS AND METHODS}

The estimated sample size for this mono-centric randomized clinical cross-over study ${ }^{18}$ was determined to be 25 subjects $(n=25)$ calculated from a pilot study on five volunteer participants with the power of the study at $80 \%$ and $\alpha$-value of 0.05 to estimate the difference in means for the outcome variables. The study was conducted from October 2015 to March 2016 after ethical clearance from the institutional ethical clearance committee as per the ethical principles of the World Medical Association (WMA)-Declaration of Helsinki and Good Clinical Practice (GCP) guidelines. The trial was registered with the clinical trials registry of India (CTRI/2018/04/013503). Eighty dental student volunteers were screened and 25 participants meeting inclusion criteria of the study were recruited by convenience sampling.

The inclusion, exclusion and withdrawal criteria for the study were as follows:

\section{Inclusion Criteria}

- Systemically healthy volunteers above 18 years of age

- Each participant having a minimum of 20 teeth with intact periodontium excluding third molars

- Each participant having good oral hygiene with a mean Pl score $<1$ (Sillness and Loe). ${ }^{19}$

\section{Exclusion Criteria}

- Participants with gingival or periodontal disease.

- Participants who had received periodontal treatment in the past 6 months.

- Participants wearing orthodontic or prosthetic appliances.

- Participants currently on antimicrobials or analgesics.

- Participants who reported tobacco use in any form.

- Participants with an allergy to constituents in dentifrice used in the study.

- Participants with mouth breathing and occlusal parafunction
- Withdrawal Criteria

- Participants could withdraw from the study as per their will

The 25 participants comprising of 18 females and 7 males all aged between 18 years to 25 years with a mean age of 22.5 years were recruited in the study. After procuring informed consent, the participants underwent ultrasonic scaling. ${ }^{8,18}$ The study plan comprised of two phases of six weeks duration ${ }^{13}$ each with assessments performed at baseline, 3 weeks and 6 weeks. A washout period of two weeks was observed between the two phases of the study (Flow chart 1).

At baseline of phase 1 visit the participants were asked to report without brushing or consuming meals to the dental office, allowing an overnight buildup of dental plaque for 12 hours. ${ }^{7,20}$ The participants were randomly allocated by computerized random allocation to two groups A (13 subjects) and B (12 subjects) which would receive the two intervention toothbrushes in a cross over study design. The participants in groups A and $B$ were then assigned and provided either of the two intervention toothbrushes by a coin toss: charcoal infused bristle toothbrush (T1) (Colgate SlimSoft ${ }^{\mathrm{TM}}$ Charcoal. Colgate-Palmolive Company, Mumbai, India) or nylon bristle toothbrush (T2) (Colgate SlimSoft ${ }^{\mathrm{TM}}$ ColgatePalmolive, Mumbai, India) (Fig. 1A). Apart from the only difference of charcoal infused bristles of Toothbrush T1 as compared to regular nylon bristles of toothbrush T2, both toothbrushes $\mathrm{T} 1$ and $\mathrm{T} 2$ were identical in all aspects of toothbrush head design, brushing plane , bristle material, bristle dimensions of length, tip diameter $(0.01 \mathrm{~mm})$, taper and arrangement within square tuft slots in the toothbrush head (Figs 1B and C). A fluoridated dentifrice containing 1000 ppm of fluoride [Colgate ${ }^{\circledR}$ Dental Cream Toothpaste Colgate-Palmolive, Mumbai, India) was provided for the duration of the study. The toothbrush wear index score (WI $)^{21}$ of toothbrushes $\mathrm{T} 1$ and $\mathrm{T} 2$ were recorded prior to dispensing the toothbrushes $\mathrm{T} 1$ and T2 to the participants by a blinded examiner according to the method of Rawls et al. ${ }^{21-23}$ The measurements for the toothbrush wear index were made by an electronic vernier caliper (Mitutoyo, Japan) (Fig. 1A) and WI was calculated by totaling of the difference in between the brushing plane level length (BPL) and head level length (HLL) plus the difference in brushing plane (BPW) and head level width (HLW) of the bristles divided by the height of the bristles (HTB), i.e., [WI $=$ [(BPL-HLL) + (BPW-HLW) $\div$ HTB] (Fig. 2). ${ }^{21-23}$ During the same baseline appointment, the participants were asked to brush their teeth in the dental office using the assigned toothbrush $\mathrm{T} 1$ or $\mathrm{T} 2$ with the fluoridated dentifrice for 2 minutes using the modified bass technique under the supervision of a non-participating examiner to see that oral hygiene 
Flow Chart 1: Study plan

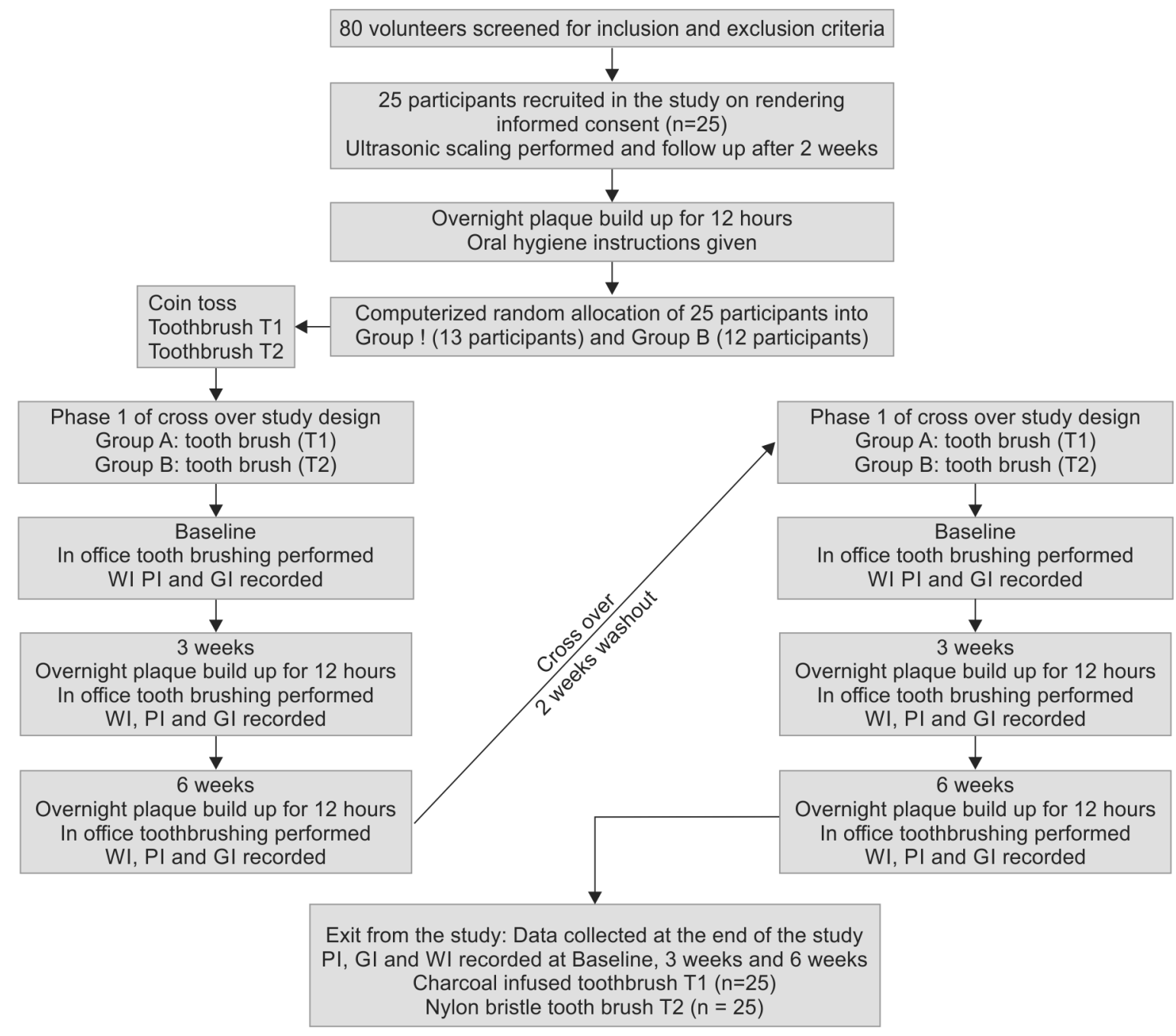

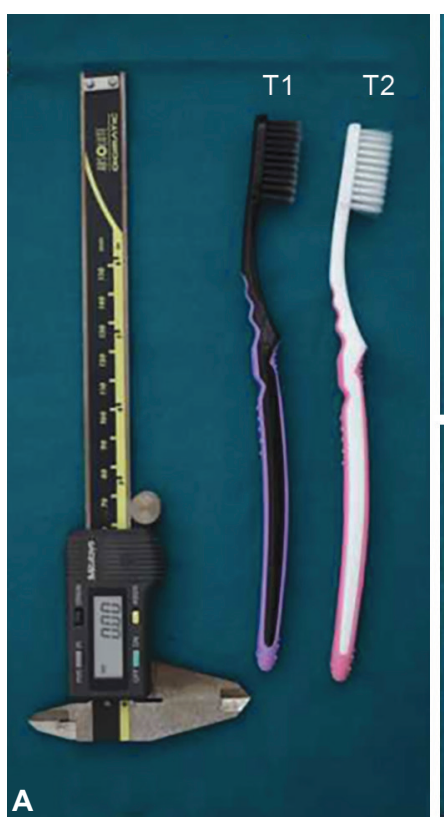
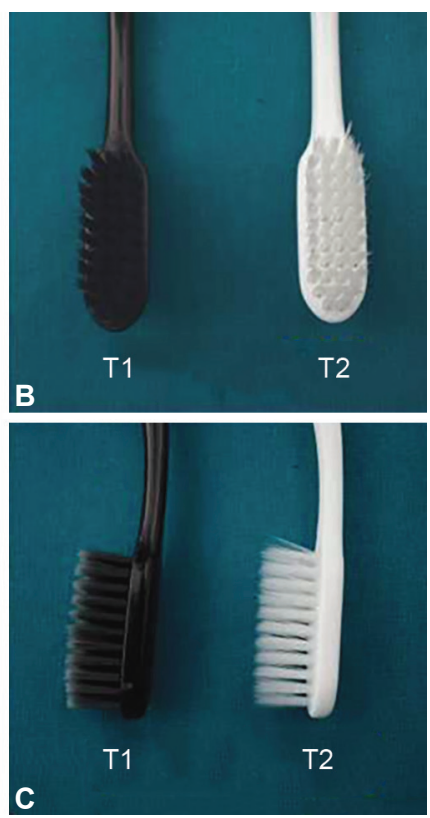

Figs 1A to C: (A) Electronic Vernier caliper and Toothbrushes T1 and T2; (B and C) T1 and T2 similar in all aspects of bristle dimension and arrangement

instructions are performed as per instructions. ${ }^{24}$ Another blinded examiner recorded a full mouth PI score

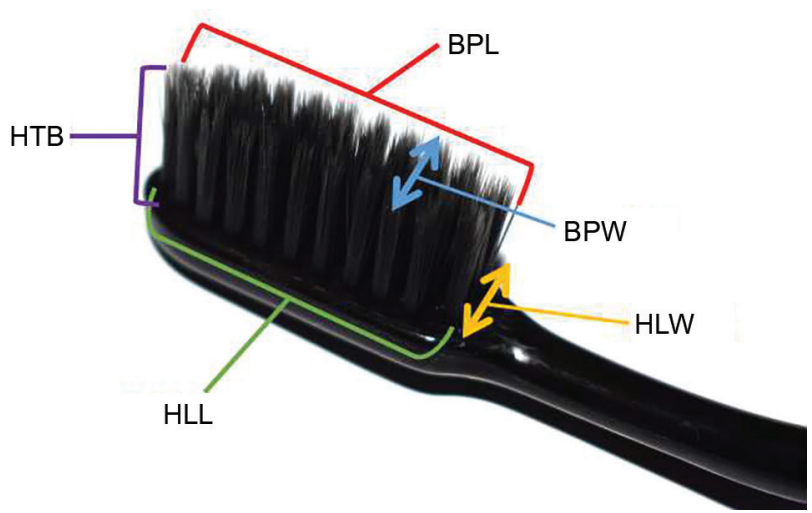

Fig. 2: Measurements for Wear index: BPL: brushing plane length, BPW: brushing plane width, HTB: height of bristle, HLW: head level width, HLL:Head level length.

$(\text { Loe })^{19}$ to evaluate remnant plaque after the in-office toothbrushing following overnight plaque buildup, and a full mouth Gingival index score (GI) (Loe et al. ) $^{25}$ for each participant. The recorded WI, PI, GI and scores at this examination were taken as the Baseline assessment. In between baseline to assessments at 3 weeks and 6 weeks, the participants were given oral hygiene instructions to brush twice daily with the assigned toothbrush and 
dentifrice with the modified Bass technique for 2 minutes at morning and night after meals throughout the study.

During the first phase of this cross over study group A received the toothbrush $\mathrm{T} 1$ whereas group $\mathrm{B}$ received the toothbrush T2. The Participants were monitored through interview weekly to ensure compliance with the oral hygiene instructions. At 3 weeks from baseline and at 6 weeks from baseline of each phase of the study WI, PI, and GI scores were recorded for each participant in the same manner as performed at baseline. The participants would bring the assigned toothbrush they were using in the period between assessments for calculation of the WI. Participants were required to report following 12 hours overnight plaque buildup refraining from brushing or consuming any meal at each assessment to record remnant plaque (PI) following in-office toothbrushing. Data for each participant was collected from the blinded examiners by a non-participating examiner. As the color of the bristles of both toothbrush T1 and T2 were distinct and gave away the type of the toothbrush under study in both groups A and B, participant blinding was not possible. Computerized random allocation was therefore performed allotting participants to each group $\mathrm{A}$ or $\mathrm{B}$ as to minimize the Hawthorne effect during both phases of the study. Following the end of the 6-week assessment of the first phase of the study, the participants were asked to revert to their previous oral hygiene practices during a washout period of two weeks. Following the washout period in the second phase of the study Group A was allocated the toothbrush T2 while group B was allocated toothbrush T1. The same study plan as in the first phase of the study was followed with WI, PI and GI recorded at baseline, 3 weeks from baseline and at 6 weeks from baseline. The study concluded uneventfully with no dropouts or reported adverse events. The data collected from both phases of the the study for WI, PI, GI and at baseline, 3 weeks from baseline and 6 weeks from baseline from 25 participants $(n=25)$ subjected to the both intervention toothbrushes T1 and T2 under study were tabulated in Microsoft Excel sheet and provided to a blinded statistician for statistical analysis.

\section{Statistical Methods}

Statistical analysis was performed by a blinded statistician using statistical analysis $\mathrm{R}$ software package version 3.5.1. The ordinal data for PI and GI and the numerical data for WI was found to be normally distributed for both toothbrush T1 and toothbrush T2 using the KolmogorovSmirnov test. The mean values with standard deviation for the parameters of PI, GI, and WI at baseline, 3 weeks from baseline and 6 weeks from baseline were generated for descriptive statistics (Table 1). Analytical statistics for the inter-group comparison of mean values for PI, GI, and WI at baseline, 3 weeks from baseline and 6 weeks from baseline for both the toothbrush $\mathrm{T} 1$ and the toothbrush $\mathrm{T} 2$ was performed using the unpaired t-test (Table 2). Intra-group comparison of the difference in means for PI, GI and WI from baseline to 3 weeks, and from 3 weeks to 6 weeks for both toothbrushes T1 and T2 was performed using the paired t-test (Table 3). A $p$ value of less than 0.05 was considered statistically significant.

\section{RESULTS}

At Baseline the mean WI for T1 $(0.049 \pm 0.018)$ and T2 $(0.40 \pm 0.017)$ (Table 1 and Graph 1) on inter-group comparison demonstrated statistically non-significant difference $(0.008778, p=0.084)$ (Table 2). At baseline the mean PI for T1 $(0.0380 \pm 0.012)$ and T2 (0.357 \pm 0.013$)$ (Table 1) on inter-group comparison demonstrated statistically higher PI for T1 compared to T2 (0.02304, $p=5.30 \mathrm{E}-08$ ) (Table 2). The mean GI scores at baseline for T1 $(0.280 \pm 0.012)$ and T2 (0.284 \pm 0.011$)$ (Table 1 and Graph 2) demonstrated statistically non significant difference on inter-group comparison $(-0.00384, p=0.239)$ (Table 2).

From Baseline to 3 weeks on intragroup comparison of $\mathrm{T} 1$ had demonstrated significant increase in WI $(0.049 \pm 0.018$ to $0.087 \pm 0.028, \mathrm{p}=4.783 \mathrm{E}-10)$ (Tables 1

Table 1: Descriptive data for the parameters of $\mathrm{WI}, \mathrm{PI}$ and $\mathrm{GI}$ at assessments for $\mathrm{T} 1$ and $\mathrm{T} 2$

\begin{tabular}{|c|c|c|c|c|c|}
\hline Assessment & Parameter & $\begin{array}{l}\text { Charcoal infused } \\
\text { bristletoothbrush (T1) } \\
n=25\end{array}$ & & $\begin{array}{l}\text { Nylon Bristle } \\
\text { toothbrush (T2) } \\
n=25\end{array}$ & \\
\hline & & Mean & Standard deviation & Mean & Standard deviation \\
\hline \multirow[t]{3}{*}{ Baseline } & WI & 0.049 & 0.018 & 0.040 & 0.017 \\
\hline & $\mathrm{PI}$ & 0.380 & 0.012 & 0.357 & 0.013 \\
\hline & $\mathrm{GI}$ & 0.280 & 0.012 & 0.284 & 0.011 \\
\hline \multirow[t]{3}{*}{3 weeks from baseline } & WI & 0.087 & 0.028 & 0.055 & 0.018 \\
\hline & $\mathrm{PI}$ & 0.353 & 0.012 & 0.343 & 0.011 \\
\hline & $\mathrm{GI}$ & 0.260 & 0.012 & 0.260 & 0.012 \\
\hline \multirow[t]{3}{*}{6 weeks from baseline } & $\mathrm{WI}$ & 0.180 & 0.075 & 0.235 & 0.053 \\
\hline & $\mathrm{PI}$ & 0.320 & 0.011 & 0.337 & 0.012 \\
\hline & $\mathrm{Gl}$ & 0.247 & 0.010 & 0.253 & 0.015 \\
\hline
\end{tabular}


Plaque Removal and Wear in Charcoal Infused and Nylon Bristle Toothbrushes

Table 2: Intergroup comparison between T1 and T2 for difference in mean for WI , PI and GI at assessments

\begin{tabular}{|c|c|c|c|c|c|c|}
\hline Assessment & Parameter & $\begin{array}{l}\text { Mean } \\
\text { Difference } \\
\text { T1-T2 }\end{array}$ & $t$ & $d f$ & $p$ value & Interpretation of $p$ value \\
\hline \multirow{3}{*}{ Baseline } & WI & 0.008778 & 1.765 & 48 & 0.084 & Nonsignificant \\
\hline & $\mathrm{PI}$ & 0.02304 & 6.441 & 48 & $5.30 \mathrm{E}-08^{*}$ & Significant \\
\hline & $\mathrm{GI}$ & -0.00384 & -1.192 & 48 & 0.239 & Nonsignificant \\
\hline \multirow{3}{*}{$\begin{array}{l}3 \text { weeks from } \\
\text { baseline }\end{array}$} & WI & 0.031223 & 4.676 & 48 & $2.40 \mathrm{E}-05^{*}$ & Significant \\
\hline & $\mathrm{PI}$ & 0.01044 & 3.256 & 48 & $0.002^{*}$ & Significant \\
\hline & $\mathrm{GI}$ & 0.00000 & 0 & 48 & 1.000 & Nonsignificant \\
\hline \multirow{3}{*}{$\begin{array}{l}6 \text { weeks from } \\
\text { baseline }\end{array}$} & WI & -0.05503 & -3 & 48 & $0.004^{*}$ & Significant \\
\hline & $\mathrm{PI}$ & -0.01648 & -4.953 & 48 & $9.47 \mathrm{E}-06^{*}$ & Significant \\
\hline & $\mathrm{GI}$ & -0.0056 & -1.577 & 48 & 0.121 & NonSignificant \\
\hline
\end{tabular}

*signifies $p<0.05$ E: Exponential

Table 3: Intragroup comparison for T1 and T2 between assessments for parameters of WI, $\mathrm{PI}$ and GI

\begin{tabular}{|c|c|c|c|c|c|c|}
\hline Toothbrush & Parameter & Assessment & Paired t-test value & $d f$ & $p$ value & Interpretation of $p$ value \\
\hline \multirow{6}{*}{$\begin{array}{l}\text { Charcoal Infused } \\
\text { BristleToothbrush } \\
\text { (T1) } n=25\end{array}$} & \multirow[t]{2}{*}{$\mathrm{WI}$} & Baseline-3 weeks & -10.014 & 24 & $4.783 \mathrm{E}-10^{*}$ & Significant \\
\hline & & 3 weeks-6weeks & -6.128 & 24 & $2.489 \mathrm{E}-6^{*}$ & Significant \\
\hline & \multirow[t]{2}{*}{$\mathrm{PI}$} & Baseline-3 weeks & 11.646 & 24 & $2.319 \mathrm{E}-11^{*}$ & Significant \\
\hline & & 3 weeks -6 weeks & 14.987 & 24 & $1.107 \mathrm{E}-13^{*}$ & Significant \\
\hline & \multirow[t]{2}{*}{ GI } & Baseline-3 weeks & 18.119 & 24 & 1.663E-15* & Significant \\
\hline & & 3 weeks-6weeks & 9.844 & 24 & $6.672 \mathrm{E}-10^{*}$ & Significant \\
\hline \multirow{6}{*}{$\begin{array}{l}\text { Nylon Bristle } \\
\text { toothbrush [T2] } \\
n=25\end{array}$} & \multirow[t]{2}{*}{ WI } & Baseline-3 weeks & -14.502 & 24 & $2.260 \mathrm{E}-13^{*}$ & Significant \\
\hline & & 3 weeks -6 weeks & -16.702 & 24 & $1.023 \mathrm{E}-14^{*}$ & Significant \\
\hline & \multirow[t]{2}{*}{ PI } & Baseline-3 weeks & 10.155 & 24 & $3.639 \mathrm{E}-10^{*}$ & Significant \\
\hline & & 3 weeks -6 weeks & 2.975 & 24 & $0.007^{*}$ & Significant \\
\hline & \multirow[t]{2}{*}{ GI } & Baseline-3 weeks & 6.709 & 24 & $6.130 \mathrm{E}-7^{*}$ & Significant \\
\hline & & 3 weeks-6 weeks & 4.508 & 24 & $1.451 \mathrm{E}-4^{*}$ & Significant \\
\hline
\end{tabular}

*signifies $p<0.05$ E: Exponential

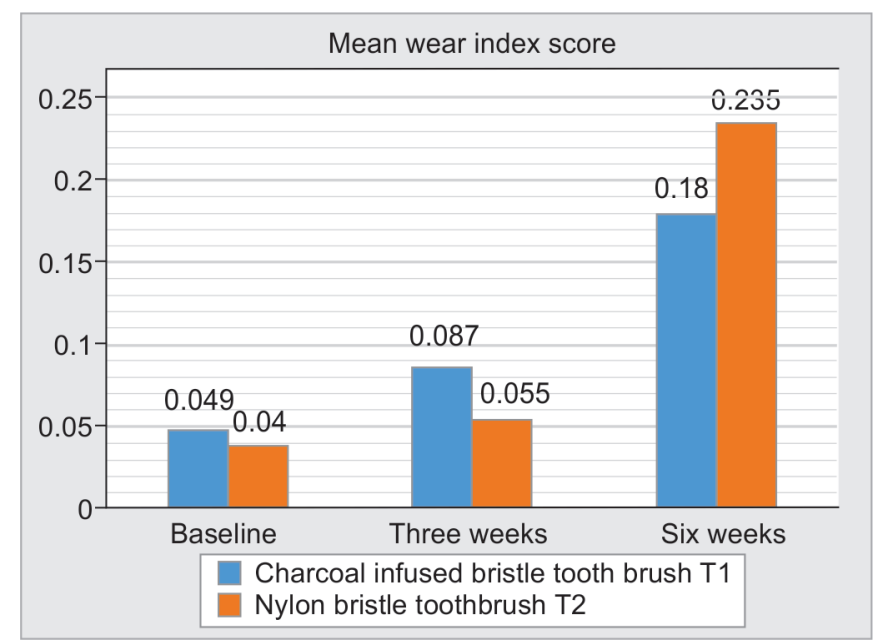

Graph 1: Mean Wear Index (WI) scores for Toothbrushes $\mathrm{T} 1$ and $\mathrm{T} 2$ at Baseline, at 3 weeks and at 6 weeks

and 3 and Graph 1), significant reduction in PI $(0.0380 \pm$ 0.012 to $0.353 \pm 0.012, p=2.319 \mathrm{E}-11$ ) (Tables 1 and 3 and Graph 2) and significant reduction in GI ( $0.280 \pm 0.012$ to $0.260 \pm 0.012, p=1.663 \mathrm{E}-15)$ (Tables 1 and 3 and Graph 3). Similarly from Baseline to 3 weeks on intra-group comparison of $\mathrm{T} 2$ had demonstrated significant increase in WI $(0.040 \pm 0.017$ to $0.055 \pm 0.018, p=2.260 \mathrm{E}-03)$ (Tables 1

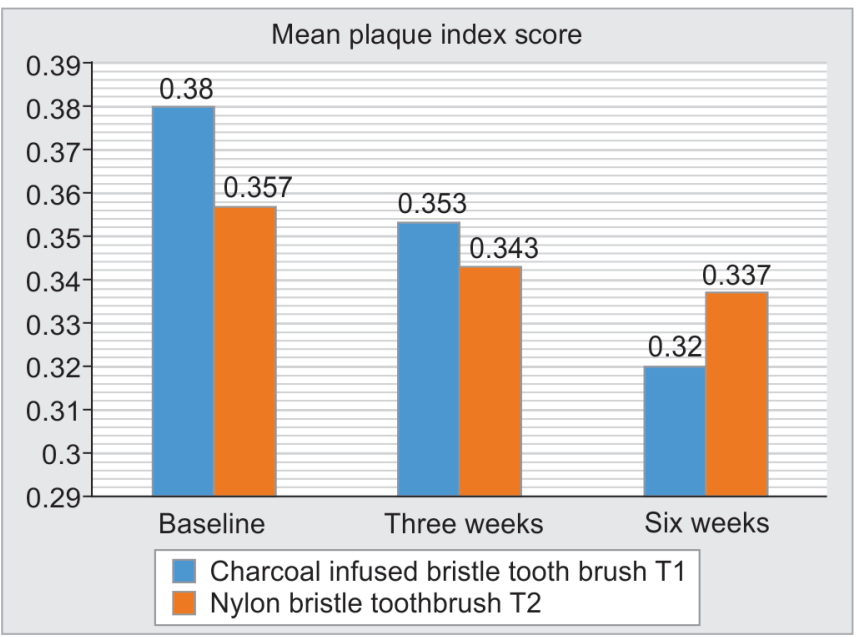

Graph 2: Mean Plaque Index (PI) scores for Toothbrushes T1 and T2 at Baseline, at 3 weeks and at 6 weeks

and 3 and Graph 1$)$, significant reduction in PI $(0.357 \pm 0.013$ to $0.343 \pm 0.011, p=3.639 \mathrm{E}-10$ ) (Tables 1 and 3 and Graph 2) and significant reduction in GI $(0.284 \pm 0.011$ to $0.260 \pm$ $0.012, p=6.130 \mathrm{E}-7$ ) (Tables 1 and 3 and Graph 3). Intergroup comparison at 3 weeks from baseline between difference in means of $\mathrm{T} 1$ and T2 (Table 2) demonstrated statistically significant higher WI $(0.031223, p=2.40 \mathrm{E}-05)$ 


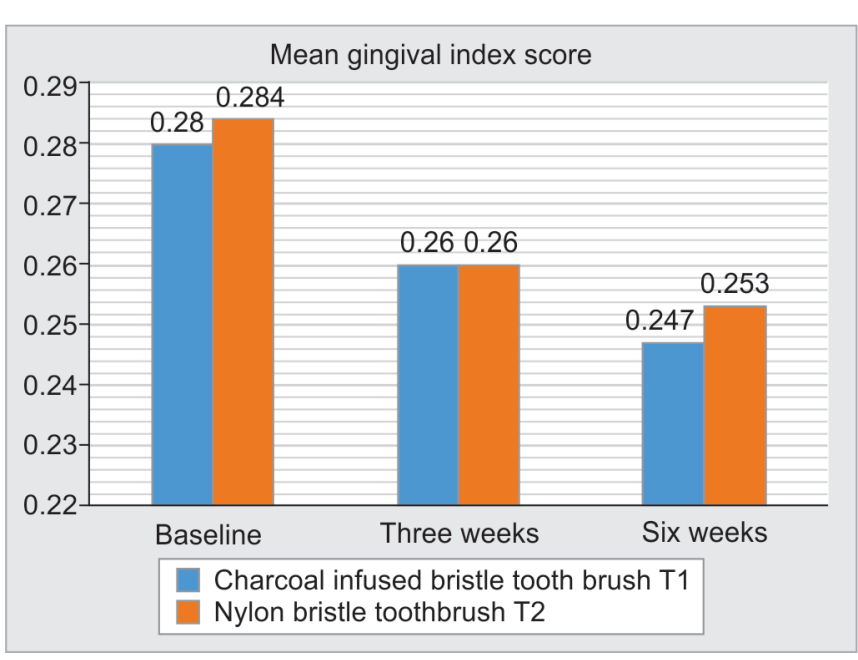

Graph 3: Mean Gingival Index [GI] scores for Toothbrushes T1 and T2 at Baseline, at 3 weeks and at 6 weeks

for T1 compared to T2, statistically significant higher PI $(0.01044, p=0.002)$ for $\mathrm{T} 1$ compared to $\mathrm{T} 2$, and statistically non-significant difference in GI $(0.00000, p=1.000)$ between $\mathrm{T} 1$ and $\mathrm{T} 2$.

From 3 weeks to 6 weeks on intra-group comparison of T1 had demonstrated significant increase in WI $(0.087 \pm 0.028$ to $0.180 \pm 0.075, p=2.489 \mathrm{E}-6)$ (Tables 1 and 3 and Graph 1), significant reduction in PI $(0.0353 \pm 0.012$ to $0.320 \pm 0.011, p=1.107 \mathrm{E}-13$ ) (Tables 1 and 3 and Graph 2) and significant reduction in GI $(0.260 \pm 0.012$ to $0.247 \pm 0.010$, $p=6.672 \mathrm{E}-10$ ) (Tables 1 and 3 and Graph 3). Similarly from 3 weeks to 6 weeks on intra-group comparison of T2 had demonstrated significant increase in WI (0.055 \pm 0.088 to $0.235 \pm 0.053, p=1.023 \mathrm{E}-14$ ) (Tables 1 and 3 and Graph 1), significant reduction in PI $(0.343 \pm 0.011$ to $0.337 \pm 0.012, p=0.007$ ) (Tables 1 and 3 and Graph 2) and significant reduction in GI ( $0.260 \pm 0.012$ to $0.253 \pm$ $0.015, p=1.451 \mathrm{E}-4)$ (Tables 1 and 3 and Graph 3). Intergroup comparison at 6 weeks from baseline between difference in means of T1 and T2 (Table 2) demonstrated statistically significant lower WI $(-0.05503, p=0.004)$ for T1 compared to T2, statistically significant lower PI (-0.01648, $p=9.47 \mathrm{E}-06)$ for T1 compared to T2, and statistically non-significant difference in GI $(-0.0056, p$ $=0.121$ ) between $\mathrm{T} 1$ and $\mathrm{T} 2$.

\section{DISCUSSION}

The aim of this study was to evaluate and compare the plaque removal efficacy and toothbrush bristle wear following use over 6 weeks of two toothbrushes $\mathrm{T} 1$ and T2 similar in all aspects of brush design and bristle dimensions with the only difference being that the bristles of T1 were charcoal infused as compared to the regular nylon bristles of T2. The study was conducted as a cross-over study so that the participants served as their controls to keep participant dexterity and brushing force similar during both phases of the study. ${ }^{2-6}$ As participants couldn't be blinded to the intervention Toothbrushes T1 and T2 due to the color of the bristles and since the participants were dental student volunteers who would be monitored on a weekly basis during both phases of the study there was a possibility of the Hawthorne effect influencing the outcomes. ${ }^{26}$ Therefore this study was planned so that the 25 participants were allocated to groups A and B using either of the two study toothbrushes T1 and $\mathrm{T} 2$ at a given point of the study phases to reduce the influence of the Hawthorne effect being confined to a single study toothbrush. ${ }^{26}$ Although its recommended to replace a toothbrush every 3 months, ${ }_{1}^{14}$ present evidence does not provide us with a time-bound parameter for replacement of toothbrush due to loss of efficacy in the removal of plaque. ${ }^{2,22}$ This study was conducted with each toothbrush used over a period of 6 weeks during each phase of the study ${ }^{9,13}$ with assessments for WI, PI and GI being performed at baseline, 3 weeks from baseline and 6 weeks from baseline. In this study oral hygiene instructions were given to all participants to adhere to similar technique, duration, and frequency so as to subject both toothbrushes to similar patterns of use which would have an influence on the wear of the bristles. A 12 hours overnight plaque buildup and preappointment abstinence from toothbrushing or consuming meals during assessments at baseline, 3 weeks and 6 weeks so that the time duration to allow plaque to accumulate was standardized for all participants not creating a bias during evaluation while recording remnant plaque through PI following in-office toothbrushing as it allowed plaque to accumulate over a fixed time of 12 hours for all participants. ${ }^{7,22}$ The modified Bass technique was chosen for this study as the soft texture bristle tips removed plaque from the cervical area of teeth along the free gingival margin and facilitated intra-sulcular brushing without causing abrasive trauma to the gingiva. $15,16,27$ In this study none of the participants reported of any incident of gingival abrasion during the entire course of the study while using either of T1 or T2. Literature does suggest that 0.2 $\mathrm{mm}$ bristle filaments effectively removed plaque better as compared to narrower diameter bristle filaments while brushing with the Bass technique. ${ }^{9,16,27}$ Both T1 and T2 had the same bristle tip diameter of $0.01 \mathrm{~mm}$; therefore, their comparison with bristle diameter of 0.2 $\mathrm{mm}$ was beyond the scope of this study. In this study, the inter-group difference in WI at baseline was not statistically significant $(p=0.084)$ indicating that the new toothbrushes $\mathrm{T} 1$ and $\mathrm{T} 2$ bristles displayed no wear during dispensing at the start of the study. The intergroup difference in PI was higher for T1 as compared 
to T2 ( $p=5.30 \mathrm{E}-08)$ indicating that the plaque removal was better at baseline for T2 as compared to T1. This can be attributed to the Hawthorne effect as the volunteers could have possibly brushed more rigorously with the colorless nylon bristle toothbrush as blinding was not possible. The supervised office oral hygiene routine was performed to limit this effect. The Inter-group difference in GI between T1 and T2 at baseline was not statistically significant $(p=0.239)$ indicating that the gingiva was at a comparable condition of health at the commencement of the study. From Baseline to 3 weeks T1 on intra-group comparison demonstrated significant increase in WI $(p=4.783 \mathrm{E}-10)$, reduction in PI $(\mathrm{p}=2.319 \mathrm{E}-11)$ and reduction in GI scores ( $p=1.663 \mathrm{E}-15)$. While from baseline to 3 weeks T2 on intra-group comparison demonstrated a significant increase in WI ( $p=2.260 \mathrm{E}-$ $13)$, reduction in PI ( $p=3.639 \mathrm{E}-10)$ and reduction in GI scores ( $p=6.130 \mathrm{E}-7)$. At 3 weeks on the inter-group comparison between $\mathrm{T} 1$ and $\mathrm{T} 2$ reveals significantly higher WI for T1 ( $p=2.40 \mathrm{E}-05)$ and higher PI for T1 $(p=0.002)$ as compared to T2. There was no significant difference in GI between T1 and T2 at 3 weeks $(p=1.000)$. This suggested that plaque removal from baseline to 3 weeks was satisfactory to maintain the gingival health with a reduction in GI for both groups. However, T1 did demonstrate more wear as compared to T2. This is in agreement with studies of Daly et al. ${ }^{13}$ and Tan et al. ${ }^{14}$ where it was suggested that satisfactory plaque control could be achieved with worn bristle toothbrushes if motivated oral hygiene were performed. From baseline to 3 weeks T2 demonstrated better plaque removal as compared to $\mathrm{T} 1$.

From 3 weeks to 6 weeks T1 on intra-group comparison demonstrated significant increase in WI ( $p=2.489 \mathrm{E}-6)$, reduction in PI ( $p=1.107 \mathrm{E}-13)$ and reduction in GI scores $(p=6.672 \mathrm{E}-10)$. While from baseline to 3 weeks T2 on intra-group comparison demonstrated significant increase in WI ( $p=1.023 \mathrm{E}-14)$, reduction in PI $(p=0.007)$ and reduction in GI scores $(p=1.451 \mathrm{E}-4)$.

At 6 weeks on the inter-group comparison between $\mathrm{T} 1$ and T2 reveals significantly lower WI for T1 $(p=0.004)$ and lower PI for T1 ( $p=9.47 \mathrm{E}-06)$ as compared to T2. There was no significant difference in GI between T1 and T2 at 6 weeks $(p=0.121)$. This suggested that plaque control from 3 weeks to 6 weeks was satisfactory to maintain the gingival health with a reduction in GI for both groups in agreement with Daly et al. ${ }^{13}$ and Tan et al. ${ }^{14}$ However at 6 week endpoint of the study T2 demonstrated more wear as compared to T1, and T1 demonstrated better plaque removal as compared to T2. This was in agreement with studies of Kreifeldt et al. ${ }^{28}$ and Glaze and Wade. ${ }^{29}$ that suggest that plaque removal efficiency reduces with bristle wear during use.

\section{LIMITATIONS}

The Haw thorne effect due to the study participants being dental student volunteers could have had an influence on the study outcomes. Daily frequency and duration of toothbrushing which could have impacted bristle wear was not monitored apart from volunteered information recorded during a weekly interview. A larger sample size comprising participants with different periodontal disease conditions of gingivitis and periodontitis could determine whether study outcomes could have therapeutic implications over patient categories with different plaque control needs.

\section{CONCLUSION}

Within the limitations of the current study setting it may be permissible to conclude that charcoal infused bristles displayed less wear and demonstrated better plaque removal efficacy as compared to plain nylon bristles of similar dimensions the following use over a period of 6 weeks.

\section{CLINICAL SIGNIFICANCE}

The results from this study show that charcoal infused nylon bristles demonstrate less wear under similar conditions of use as compared to nylon bristles having the same design and dimensions. Whether these results are observed over a longer time span of use in larger populations having different plaque control needs is subject to further research.

\section{REFERENCES}

1. Breuer MM, Cosgrove RS. The Relationship Between Gingivitis and Plaque Levels. J. Periodontol.1989; 60(4):172-175.

2. Asadoorian J. CDHA. Position Paper on Tooth Brushing. CJDH. 2006; 40(5): 232-248.

3. Bergenholtz A, Gustafsson LB, Segerlund N, et al. The role of brushing technique and toothbrush design in plaque removal. Scand J Dent Res 1984;92(4):344-351.

4. Tere'zhalmy GT, Biesbrock AR, Walters PA, et al. Clinical evaluation of brushing time and plaque removal potential of two manual toothbrushes. Int J Dent Hygiene. 2008;6(4):321327.

5. Van der Weijden GA, Timmerman MF, et al. Relationship between the plaque removal efficacy of a manual toothbrush and brushing force. J Clin Periodontol. 1998; 25(5):413-416.

6. Nieri M, Giani M, Pagliaro U, et al. Efficacy and preference of manual toothbrushes: a randomised, single blind, controlled trial. Eur J Oral Implantol 2013;6(2):181-188.

7. Harpenau L, Meyers G, Lyon C, et al. Blinded Clinical Evaluation of a New Manual Toothbrush. J Clin Dent. 2006; 17 (1):1-4.

8. Claydon N, Addy M, Scratcher C, et al. Comparative professional plaque removal study using 8 branded toothbrushes. J Clin Periodontol. 2002;29(4):310-316. 
9. Hedge S, Kakade AA, Rajesh KS, et al. Evaluation of plaque removal efficacy of two manual toothbrushes with different textures: a comparative analysis. Journal of Oral Health Research. 2011; 2(3):84-90.

10. Claydon N, AddyM. Comparative single-use plaque removal by toothbrushes of different designs. J Clin Periodontol. 1996; 23 (12): 1112-1116.

11. DörferCE, von Bethlenfalvy ER, Kugel B, et al. Cleaning Efficacy of a Manual Toothbrush with Tapered Filaments. Oral Health Prev Dent 2003;1(2):111-118.

12. Tangade PS, Shah AF, Ravishankar TL, et al. Pal S. Is plaque removal efficacy of toothbrush related to bristle flaring? A 3-month prospective parallel experimental study. Ethiop J Health Sci. 2013; 23(3):255-264.

13. Daly CG, Chappie CC, Cameron AC. Effect of toothbrush wear on plaque control.J Clin Periodontol 1996:23(1):45-49.

14. Tan E, Daly C. Comparison of new and 3-month-old toothbrushes in plaque removal. J Clin Periodontol. 2002; 29 (7): 645-650.

15. Versteeg PA, Rosema NAM, Timmerman MF, et al. Evaluation of two soft manual toothbrushes with different filament designs in relation to gingival abrasion and plaque removing efficacy. Int J Dent Hygiene. 2008;6(3):166-173.

16. Vowles AD, Wade AB. Importance of Filament Diameter when Using Bass Brushing Technique. J Periodontol.1977; 48(8):460-463.

17. Cifcibasi E, Koyuncuoglu CZ, Baser U, Bozacioglu B, et al. Comparison of manual toothbrushes with different bristle designs in terms of cleaning efficacy and potential role on gingival recession. Eur J Dent 2014;8(3):395-401.

18. McCracken GI, Steen N, Preshaw PM, et al. The crossover design to evaluate the efficacy of plaque removal in tooth-brushing studies. J Clin Periodontol 2005; 32 (11): 1157-1162.

19. Loe H. The Gingival Index, the Plaque Index and the Retention Index Systems. J Periodontol.1967;38(6):610-616.

20. Van der Weijden GA, Timmerman MF, Piscaer M, et al. Effectiveness of an electrically active toothbrush in the removal of overnight plaque and treatment of gingivitis. J Clin Periodontol.2002;29(8):699-704.

21. Rawls HR, Mkwayi-Tulloch NJ, Casella R, et al. The Measurement of Toothbrush Wear. J Dent Res. 1989; 68(12):1781-1785.

22. Rawls HR, Casella R, Mkwayi-Tulloch NJ. An in vitro and in vivo Study of Toothbrush bristle Splaying. J Dent Res. 1993;72(5):947-952.

23. Sforza NM, Rimondini L, di Menna F, et al. Plaque removal by worntoothbrush. J Clin Periodontol .2000; 27 (3): 212-216.

24. Williams K, Ferrante A, Dockter K, et al. One- and 3- Minute Plaque Removal by a Battery Operated Versus a Manual toothbrush. J Periodontol. 2004; 75(8):1107-1113.

25. Loe $\mathrm{H}$, Theilade E, Jensen SB. Experimental gingivitis in man. J Periodontol.1965.36(3):177-187.

26. Costa MR, da Silva VC, Miqui MN, et al. Effects of ultrasonic, electric, and manual toothbrushes on subgingival plaque composition in orthodontically banded molars. Am J Orthod Dentofacial Orthop 2010;137(2): 229-235.

27. Gibson JA, WadeAB. Plaque removal by the Bass and Roll brushing techniques. J Periodontol. 1977;48(8):456-459.

28. Kreifeldt JG, Hill PH, Calisti LJ. A systematic study of the plaque removal efficacy of worn toothbrushes. J Dent Res. 1908:59(12):2047-2055.

29. Glaze PM, Wade AB. Toothbrush age and wear as it relates to plaque control. J Clin Periodontol. 1986;13(1):52-56. 